Check for updates

Cite this: Analyst, 2019, 144, 4647

\section{Serum-based differentiation between multiple sclerosis and amyotrophic lateral sclerosis by Random Forest classification of FTIR spectra $\uparrow$}

\author{
Youssef El Khoury, (D) *a Nicolas Collongues, ${ }^{b, c}$ Jérôme De Sèze, ${ }^{b, c}$ Vildan Gulsari, ${ }^{a}$ \\ Christine Patte-Mensah, ${ }^{b}$ Gilles Marcou, (iD d Alexandre Varnek, id d \\ Ayikoé Guy Mensah-Nyagan (D b and Petra Hellwig (iD *a,e
}

\begin{abstract}
The challenging diagnosis and differentiation between multiple sclerosis and amyotrophic lateral sclerosis relies on the clinical assessment of the symptoms along with magnetic resonance imaging and sampling cerebrospinal fluid for the search of biomarkers for either disease. Despite the progress made in imaging techniques and biomarker identification, misdiagnosis still occurs. Here we used $2.5 \mu \mathrm{L}$ of serum samples to obtain the infrared spectroscopic signatures of sera of multiple sclerosis and amyotrophic lateral sclerosis patients and compared them to those of healthy controls. The spectra are then classified with the help of a two-fold Random Forest cross-validation algorithm. This approach shows that infrared spectroscopy is powerful in discriminating between the two diseases and healthy controls by offering high specificity for multiple sclerosis (100\%) and amyotrophic lateral sclerosis (98\%). In addition, data after six and twelve months of treatment of the multiple sclerosis patients with biotin are discussed.
\end{abstract}

Received 25th April 2019, Accepted 6th June 2019 DOI: $10.1039 /$ c9an00754g rsc.li/analyst lated syndrome, relapsing-remitting, and secondary progressive or primary progressive. ${ }^{1}$ ALS is characterized by the progressive and inevitable death of motor neurons. ${ }^{2}$ MS and ALS share similar symptoms especially in the early phase which makes their diagnosis and differentiation a challenging and a time-consuming task.

The diagnosis of the aforementioned conditions mainly relies on the interpretation of clinical symptoms on the one hand, and on the other hand on magnetic resonance imaging (MRI) as well as cerebrospinal fluid (CSF) sampling in search for disease biomarkers. ${ }^{3-6}$ Despite the advances in imaging witnessed over the last few decades, misdiagnosis still occurs and sometimes involves $10 \%$ of the examined patients. ${ }^{7}$ The misdiagnosis is caused by several factors, for instance the resemblance between the symptoms of different neurodegenerative diseases as well as the clinical and pathophysiological complexities in a single disease such as MS where four different phenotypes exist. Misdiagnosis can unfortunately lead to a compromise in patients' lives instead of improving their well-being. Several criteria and guidelines for the diagnosis of $\mathrm{MS}^{8}$ and $\mathrm{ALS}^{9}$ were introduced in order to prevent misdiagnosis. Yet, some criteria are never fulfilled to make a clearcut diagnosis; for example, the brain MRI of ALS patients is normal at the onset of the disease and $10 \%$ of ALS patients never fulfill all the criteria for "ALS positive". ${ }^{10}$ This example illustrates the unmet need for providing an unambiguous diagnosis. In addition, patients with a pacemaker cannot be exam- 
ined by MRI and the same goes for claustrophobic patients. Besides the misdiagnosis problem, both MRI and CSF sampling have serious drawbacks. To overcome the limitation of MRI imaging, alternative approaches track several biomarkers that have been identified so far in the CSF as well as in blood. However, sampling the fluid from the patient's spinal cord is invasive and leakage-caused complications could occur. Unlike CSF, sampling blood is a routine procedure and can thus be considered for tracking the biomarkers. So far, the study of biomarkers in biological fluids has often involved a specific biomarker such as, for example, a particular protein, or DNA, requiring sophisticated and expensive biochemical analyses.

The need for early and reliable differential diagnosis of neurodegenerative diseases is imperative for starting advanced specific therapies as soon as possible. This has led us to use Fourier transform infrared (FTIR) spectroscopy as a methodology of diagnosis using a small volume of blood serum. Besides being an established tool in analytical chemistry, FTIR spectroscopy is being established as a tool to address key questions in biology and medicine. In particular, FTIR spectroscopy is emerging as a tool to distinguish diseased samples from healthy ones ${ }^{11-21}$ which is paving the way for FTIR spectroscopy to become a clinical diagnosis tool.

Besides the state-of-the-art clinical diagnosis of MS, new approaches for diagnosis have been investigated such as FTIR spectroscopy on the CSF samples of MS patients ${ }^{20}$ and nanotechnology-based analysis of exhaled breath. ${ }^{22}$ In this study we aim at using the major advantages of FTIR spectroscopy such as its sensitivity to small changes in the composition of an ensemble of known and unknown biomarkers of a complex fluid (e.g. serum) which provides a unique FTIR signature that can be used as a "barcode" of the disease. Moreover, the technique is reagent-free and sensitive, requires small volume samples $(2-3 \mu \mathrm{L})$, and is cost-effective since an FTIR spectrometer is affordable by most hospitals and clinics. Finally, the technique is user-friendly and fast in yielding results (in a few minutes). Our approach is to differentiate not only the sera of progressive MS patients from those of healthy controls (HC) but also those of patients with the closely related disease, ALS.

Treatment of progressive MS patients with high-dose biotin was shown to be safe and well tolerated. ${ }^{23}$ Biotin is a vitamin essential for the synthesis of fatty acids; thus it can help in the process of repairing myelin. The trial of high-dose biotin showed that $12.6 \%$ of the patients with non-active progressive MS had MS-related disability reversed. ${ }^{24}$ In order to assess the effect of the treatment on the spectra of sera of the MS patients, we included the spectra of sera after 6 and 12 months of treatment with a high dose of biotin.

\section{Materials and methods}

\section{Samples preparation}

80 serum samples from HC donors were purchased from the "Etablissement Français du sang” (Strasbourg, France). 32 serum samples of MS patients and 32 serum samples of ALS patients were prepared from whole blood collected at the "service de maladies inflammatoires du système nerveux" at Hôpital de Hautepierre in Strasbourg, France, where the patients were diagnosed using a battery of clinical and paraclinical techniques including MRI scans. The 32 MS serum samples were studied before starting the treatment (M0) with a high dose of biotin (100 mg thrice per day). After 6 months of treatment (M6), 27 serum samples were studied and after 12 months of treatment (M12) 30 serum samples were studied. The separation of serum from whole blood was conducted by centrifugation at $1500 \mathrm{~g}$ for 30 minutes at $4{ }^{\circ} \mathrm{C}$. The obtained sera were collected in plastic tubes and flash frozen in liquid nitrogen and preserved at $-80{ }^{\circ} \mathrm{C}$. Informed consent was obtained from all human subjects. Handling of the human samples is approved by the bioethics cell of the French ministry of higher education, research and innovation "Cellule Bioéthique-DGRI-SPFCO Ministère de l'enseignement supérieur, de la recherche et de l'innovation", under the reference: DC-2018-3209. The authors confirm that all experiments were performed in accordance with the relevant guidelines and regulations. Details about the cohort are given in Table 1 and Tables S1 and S2 (see the ESI $\dagger$ ).

\section{FTIR spectroscopy}

Spectra were obtained using a Vertex 70 FTIR spectrometer (Bruker Optics, Karlsruhe) equipped with a diamond ATR and liquid nitrogen-cooled MCT detector. The spectra were recorded in the $4000-700 \mathrm{~cm}^{-1}$ spectral range with a resolution of $4 \mathrm{~cm}^{-1}$ at a scan rate of $20 \mathrm{kHz}$. For each sample $2.5 \mu \mathrm{L}$ were deposited on the diamond crystal and left to dry (10 minutes). For each individual one sample was measured and at least

Table 1 Number of samples per group and their corresponding gender ratio as well as the age range, average age and standard deviation (in years). The sub-group of MS patients consists of 32 patients before treatment with biotin (M0), after 6 months of treatment (M6) and after 12 months of treatment (M12)

\begin{tabular}{|c|c|c|c|c|c|}
\hline \multirow[b]{2}{*}{ Group } & \multirow[b]{2}{*}{$\mathrm{HC}$} & \multicolumn{3}{|l|}{ MS } & \multirow[b]{2}{*}{ ALS } \\
\hline & & M0 & M6 & M12 & \\
\hline Number of samples & 80 & 32 & 27 & 30 & 32 \\
\hline Gender & $40 \mathrm{M} / 40 \mathrm{~F}$ & $11 \mathrm{M} / 21 \mathrm{~F}$ & $11 \mathrm{M} / 16 \mathrm{~F}$ & $11 \mathrm{M} / 19 \mathrm{~F}$ & $15 \mathrm{M} / 17 \mathrm{~F}$ \\
\hline Age range & $\begin{array}{l}40 \text { more than } 60 \text { years old } \\
40 \text { less than } 60 \text { years old }\end{array}$ & $38-78$ & $42-78$ & $38-78$ & $44-87$ \\
\hline Average age (years) $\pm \mathrm{SD}$ & Not available & $60.25 \pm 9.16$ & $61.29 \pm 8.64$ & $60.73 \pm 9.54$ & $64.44 \pm 10.42$ \\
\hline
\end{tabular}


5 spectra of 64 scans of each were averaged. The averaged spectra were preprocessed with OPUS 7.2 where the baseline was corrected using a single iteration polynomial rubber band function. Afterwards the spectra were normalized and the second derivative is generated. The post-processing of the data is crucial in order to avoid variations not related to the serum composition.

\section{Data analysis}

Analysis was performed using a two-fold cross-validation approach based on a Random Forest classifier algorithm ${ }^{25}$ using bagging of 100 Random Trees. We used the Weka (https://www.cs.waikato.ac.nz/ml/weka/) ${ }^{26}$ implementation, a free and open source machine learning package developed at the University of Waikato, Hamilton, New Zealand. The 144 spectra are split into a training set of 126 spectra $(70 \mathrm{HC}, 28$ M0 and 28 ALS) and a test set of 18 spectra (10 HC, 4 M0 and 4 ALS). The spectra of the test set are randomly selected while keeping the percentage of each pathological group the same in both training and test sets. The evaluation of the model is conducted by calculating the ROC (Receiver Operating Characteristic) curves as well as the precision, sensitivity and selectivity of the model towards each group of sera spectra.

The model has been calibrated on our samples and is available for download as ESI. $\dagger$ A brief explanation on how to use the model and how to recalibrate it is provided in the ESI. $\dagger$

Principal component analysis using $R^{27}$ was performed on the second derivative of the spectra but did not yield a satisfactory discrimination (see Fig. S1, S2 and S3 in the ESI†).

\section{Results and discussion}

\section{HC vs. MS and ALS}

The spectra of all the HC, M0 and ALS serum samples in the 3700-2700 and $1800-700 \mathrm{~cm}^{-1}$ regions are assembled in Fig. $1 \mathrm{~A}$ where the absorption of lipids, proteins, carbohydrates and nucleic bases occurs. ${ }^{28}$ The averaged spectra of each population allows the better observation of slight differences between the different populations (Fig. 1B). The most intense bands arise mainly from the amide modes of proteins. ${ }^{29}$ These bands do not show a significant difference from one population to the other, most likely due to the abundance of the human serum albumin protein compared to other proteins in the serum samples. These differences are better seen in the second derivatives of the averaged spectra which are shown in Fig. 1C. The assignment of the FTIR absorption bands observed here is rather challenging since serum is a very complex biofluid and contains numerous different molecules. Yet, it is known that contributions from the functional groups of biomolecules dominate certain parts of the spectrum and these contributions are summarized in Table S3 in the ESI. $\dagger$

The $3700-2700 \mathrm{~cm}^{-1}$ range of the spectra shown in Fig. 1 is the least affected one by the diseases and no significant difference can be observed. The $1700-1200 \mathrm{~cm}^{-1}$ spectral range is dominated by absorptions from proteins and lipids and shows

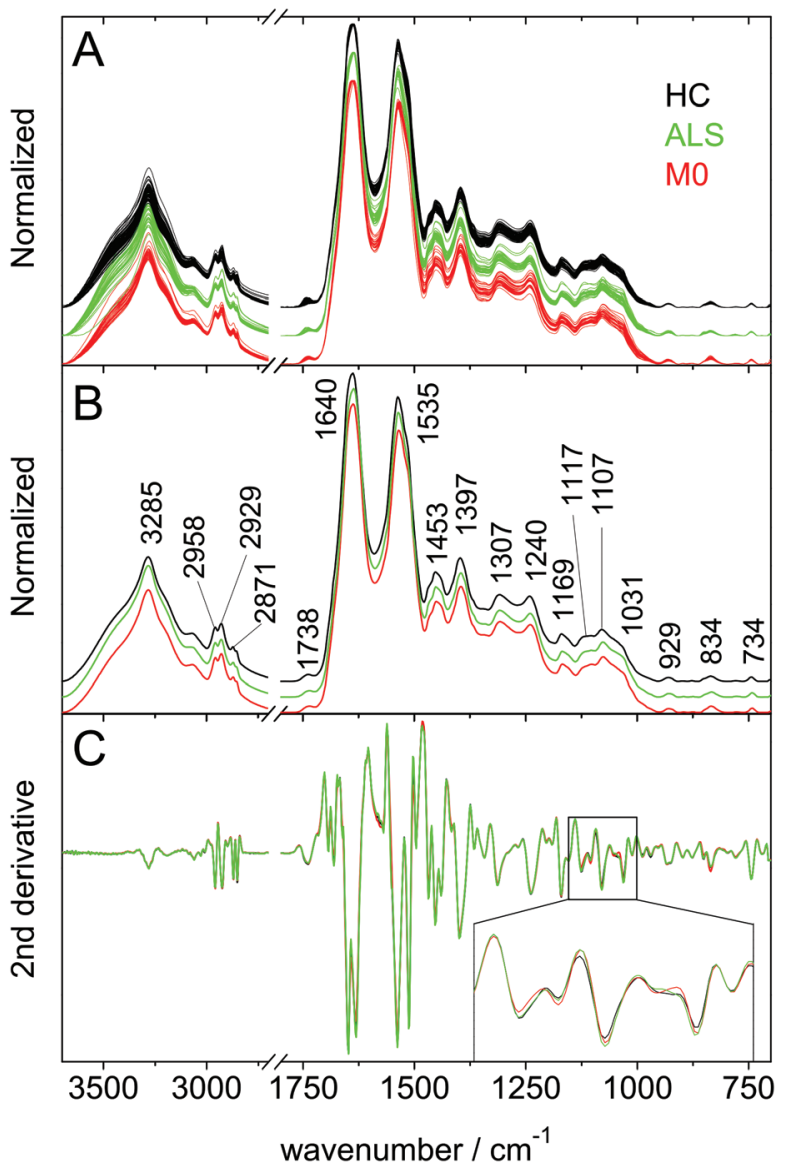

Fig. 1 (A) Normalized FTIR spectra of the serum samples in the $3700-2700$ and $1800-700 \mathrm{~cm}^{-1}$ spectral ranges. (B) Averaged spectra of the serum samples. The spectra in panels $A$ and $B$ are off-set for clarity. (C) Second derivatives of the average spectra. The inset in panel $C$ is a magnification of the data in the $1150-1000 \mathrm{~cm}^{-1}$ spectral range to highlight the slight differences between the different second derivatives. Healthy controls (black), amyotrophic lateral sclerosis (green) and multiple sclerosis (red).

slight differences between the three groups of sera. The most prominent differences are observed in the $1200-1000 \mathrm{~cm}^{-1}$ spectral range which is dominated by absorptions from carbohydrates, DNA and RNA. Accordingly, the diseases (MS and ALS) seem to modify the composition or the structure of DNA and RNA in the serum. ${ }^{30,31}$ At the same time, the affected signature of carbohydrates may reflect modifications in glycolipids, glycoproteins and collagen.

Random Forest cross-validation classification and validation. The two-fold Random Forest cross-validation is applied on 126 second derivatives of the spectra of the training set and the resulting confusion matrix is shown in Table 2. The ability of the used classifier in discriminating between the different groups of sera is confirmed by plotting the true positive rate vs. the false positive rate (ROC curves) for each group in the training set as shown in Fig. S4.† The area under the curve is minimum 95\% for any of the three groups of sera (Table 2). Among the 126 samples, 17 are misclassified leading to an 
Table 2 Confusion matrices of FTIR spectra's second derivatives of serum samples of the training (70 HC, 28 MO and 28 ALS) and test sets (10 HC, $4 \mathrm{MO}$ and $4 \mathrm{ALS}$ ) as classified using the two-fold Random Forest cross-validation algorithm along with performances metrics (ROC area, sensitivity, selectivity and precision) of the model towards each group of samples. True positives are in bold and false negative/false positive are in italics

\begin{tabular}{|c|c|c|c|c|c|c|c|c|}
\hline & \multirow[b]{2}{*}{ Pathology } & \multicolumn{3}{|c|}{ Classified as } & \multirow[b]{2}{*}{ ROC area (\%) } & \multirow[b]{2}{*}{ Sensitivity (\%) } & \multirow[b]{2}{*}{ Specificity (\%) } & \multirow[b]{2}{*}{ Precision (\%) } \\
\hline & & $\mathrm{HC}$ & MS (M0) & ALS & & & & \\
\hline & ALS & 12 & 0 & 16 & 95.0 & 57.1 & 98.0 & 88.9 \\
\hline \multirow[t]{2}{*}{ Test set } & $\mathrm{HC}$ & 10 & 0 & 0 & 88.1 & 100 & 62.5 & 76.9 \\
\hline & MS (M0) & 2 & 2 & 0 & 78.6 & 50.0 & 100 & 100 \\
\hline
\end{tabular}

overall precision of $86.5 \%$ and no HC sample among the 70 included in this study are misclassified as ALS nor as MS (M0). Therefore, there is no risk of diagnosing a healthy patient as having one of the two pathologies, MS or ALS. The sensitivity of the model towards ALS is 57.1\%. These results reflect difficulty in detecting ALS in the FTIR spectra of the serum samples; therefore patients with ALS have a high risk of being confused with HC and MS, yet the patients who are classified as ALS have a very high probability $(98.0 \%)$ of being indeed ALS patients. Similarly the model shows a sensitivity of $82.1 \%$ and a specificity of $100 \%$ towards MS. Accordingly, the high specificity of the model towards ALS and MS allows the differentiation between the two medical conditions based on the FTIR spectra of serum samples.

Using the model built here on the training test, a test set of 18 spectra not included in the training test yield the confusion matrix as shown in Table 2. The overall precision is $83.3 \%$ and is comparable to the one found for the training set. All the HC are correctly classified which confirms the power of the model in preventing a misdiagnosis of a healthy person as having either M0 or ALS. No ALS or M0 spectra were confused between each other leading to specificity values of $100 \%$ for both pathologies. The sensitivity values obtained for the test set are comparable with those of the training set.

A previous very interesting study examined the CSF samples of MS patients by FTIR spectroscopy. ${ }^{20}$ The discrimination capabilities of the two approaches are comparable where our model provides a higher specificity (100\% vs. 95\%) on the one hand and a lower sensitivity $(82.1 \%$ vs. 92\%) on the other hand. It should be noted that the sampling serum from patients is less invasive than sampling CSF. It should be noted that comparing the predictive capabilities of the present and the previously published study ${ }^{20}$ is not straightforward since the latter one deals with relapsing-remitting and clinically-isolated syndrome while the present one deals with primary and secondary progressive MS. Another recently published study based on the exhaled breath of MS patients ${ }^{22}$ shows lower predictive capabilities than the present one.

Note that the Random Forest algorithm outputs a score for each pathology on which the diagnostic decision is taken. It is possible to adjust the thresholds on these scores to clinical practices, in order to reduce the number of false positive for a given category, to the cost of increasing the number of false negative and vice versa. The high ROC area values mean that it is easy to find other compromises.

\section{M0 vs. M6 and M12}

The FTIR data in the $3700-2700$ and $1800-700 \mathrm{~cm}^{-1}$ regions of the MS serum samples before (M0) and after 6 (M6) and 12 months (M12) of treatment with a high dose of biotin are shown in Fig. 2A. The spectra of MS patients before and after

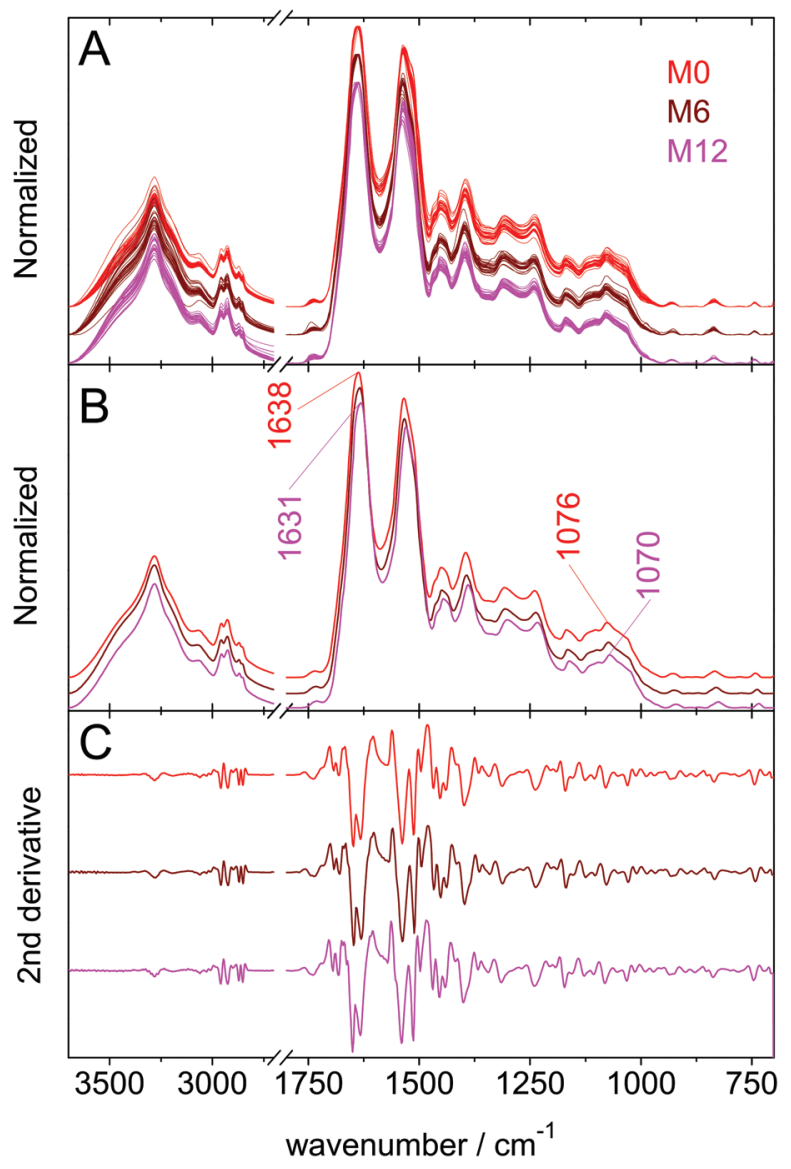

Fig. 2 (A) Normalized FTIR spectra of the serum samples in the $3700-2700$ and $1800-700 \mathrm{~cm}^{-1}$ spectral ranges. (B) Averaged spectra of the serum samples. (C) Second derivatives of the average spectra. Multiple sclerosis before (red), multiple sclerosis M6 (wine) and multiple sclerosis M12 (magenta). The spectra and second derivatives are off-set for clarity. 
Table 3 Confusion matrices of FTIR spectra's second derivatives of serum samples of the training (28 M0, 25 M6 and 27 M12) and test sets (4 M0, 2 M6 and 3 M12) as classified using the two-fold Random Forest cross-validation algorithm along with performances metrics (ROC area, sensitivity, selectivity and precision) of the model towards each group of samples. True positives are in bold and false negative/false positive are in italics

\begin{tabular}{|c|c|c|c|c|c|c|c|c|}
\hline & \multirow[b]{2}{*}{ Pathology } & \multicolumn{3}{|c|}{ Classified as } & \multirow[b]{2}{*}{ ROC area (\%) } & \multirow[b]{2}{*}{ Sensitivity (\%) } & \multirow[b]{2}{*}{ Specificity (\%) } & \multirow[b]{2}{*}{ Precision (\%) } \\
\hline & & M0 & M6 & M12 & & & & \\
\hline & M12 & 2 & 7 & 18 & 90.1 & 66.7 & 90.6 & 78.3 \\
\hline \multirow[t]{2}{*}{ Test set } & M0 & 3 & 0 & 1 & 100 & 75.0 & 100 & 100 \\
\hline & M6 & 0 & 2 & 0 & 85.7 & 100 & 85.7 & 66.7 \\
\hline
\end{tabular}

treatment show that most of the infrared signals downshift (Fig. 2B) and the downshift becomes more important when the duration of the treatment is longer. The observed downshift could be the result of the treatment itself or the progression of the disease in 6 and 12 months.

Random Forest cross-validation classification and validation. A training set (28 M0, 25 M6 and $27 \mathrm{M} 12$ ) and a test set (4 M0, 2 M6 and 3 M12) yield the confusion matrices shown in Table 3 and the ROC curves of each group in the training set are shown in Fig. $\mathrm{S} 5 \dagger$ where the area under the curve is higher than $90 \%$ for any group.

The overall precision in classifying M0, M6 and M12 is $77.5 \%$. The specificity towards M0 and M12 is high; however, the sensitivity towards M12 is $66.7 \%$. This low sensitivity shows that the model does not discriminate accurately between 6 and 12 months of treatment since 7 out of 27 of the M12 are misclassified as M6 and 2 M12 are misclassified as M0 and 2 out of the 25 M6 are misclassified as M12 and 2 as M0. The sensitivity towards M0 is high with a value of $82.1 \%$; thus, the treatment with biotin for 6 months does change the infrared signature of the serum samples of MS patients; however, after 6 months of treatment the changes stop and the signature of M12 remains very similar to that of M6. The results of the test set are in agreement with the training set.

\section{Conclusions}

Here we demonstrate the power of infrared spectroscopy coupled to Random Forest classification in discriminating between the spectra of sera of MS, ALS and HC. This rapid, reagent-free, minimally-invasive approach shows an overall high precision of $86.5 \%$ and high specificity towards MS and ALS. Accordingly the misclassification rate between the two diseases is very low. The sensitivity of this method is very high towards $\mathrm{HC}$; therefore the risk of misdiagnosis of a healthy control is very low. On the other hand, the approach is less sensitive towards MS and lesser towards ALS thus the risk of not diagnosing a MS or ALS patient with any of the two diseases is high. However, the discrimination between ALS and MS patients is good. We also show that the treatment of MS patients with high-dose biotin for 6 months changes the spectroscopic signature of the serum sample leading to a high discrimination rate between them. The impact of biotin on the spectra does not significantly change after 12 months, indicating that, on average, the patients' sera have reached a steady state.

This pilot study shows excellent features for differentiating MS and ALS serum samples and makes infrared spectroscopy a potential future tool to help prevent misdiagnosis of MS patients with ALS and vice versa. Despite the relatively small number of samples, our results advocate the use of FTIR analysis of sera as a fast, cost-effective and reliable tool for the diagnosis of MS and ALS. Finally, by making the model used here available online, other researchers can apply this model and re-calibrate it with their own data which can help to improve the overall quality of the model.

\section{Conflicts of interest}

There are no conflicts to declare.

\section{Acknowledgements}

We are grateful for the continuous support from the University of Strasbourg and the CNRS. YEK and PH are thankful to Pr. Erik Goormaghtigh for the valuable discussions. We would like to thank the Etablissement Français du Sang for the samples of the healthy controls and we are indebted to the patients who participated in this study. We are grateful to Dr Gabrielle Rudolf and Dr Marie-Céline Fleury for their continuous help and support.

\section{Notes and references}

1 F. D. Lublin, S. C. Reingold, J. A. Cohen, G. R. Cutter, P. S. Sorensen, A. J. Thompson, J. S. Wolinsky, L. J. Balcer, B. Banwell, F. Barkhof, B. Bebo Jr., P. A. Calabresi, M. Clanet, G. Comi, R. J. Fox, M. S. Freedman, A. D. Goodman, M. Inglese, L. Kappos, B. C. Kieseier, J. A. Lincoln, C. Lubetzki, A. E. Miller, X. Montalban, P. W. O'Connor, J. Petkau, C. Pozzilli, R. A. Rudick, M. P. Sormani, O. Stuve, E. Waubant and C. H. Polman, Neurology, 2014, 83, 278-286. 
2 L. H. Goldstein and S. Abrahams, Lancet Neurol., 2013, 12, 368-380.

3 A. Rovira, M. P. Wattjes, M. Tintore, C. Tur, T. A. Yousry, M. P. Sormani, N. De Stefano, M. Filippi, C. Auger, M. A. Rocca, F. Barkhof, F. Fazekas, L. Kappos, C. Polman, D. Miller, X. Montalban and M. S. group, Nat. Rev. Neurol., 2015, 11, 471-482.

4 A. Saiz, L. Zuliani, Y. Blanco, B. Tavolato, B. Giometto, F. Graus and N. M. O. S. G. Spanish-Italian, J. Neurol., 2007, 254, 1233-1237.

5 M. M. El Mendili, J. Cohen-Adad, M. Pelegrini-Issac, S. Rossignol, R. Morizot-Koutlidis, V. Marchand-Pauvert, C. Iglesias, S. Sangari, R. Katz, S. Lehericy, H. Benali and P. F. Pradat, PLoS One, 2014, 9, e95516.

6 R. Tortelli, M. Copetti, M. Ruggieri, R. Cortese, R. Capozzo, A. Leo, E. D’Errico, M. Mastrapasqua, S. Zoccolella, F. Pellegrini, I. L. Simone and G. Logroscino, Eur. J. Neurol., 2015, 22, 215-218.

7 A. J. Solomon and B. G. Weinshenker, Curr. Neurol. Neurosci. Rep., 2013, 13, 403.

8 A. J. Thompson, B. L. Banwell, F. Barkhof, W. M. Carroll, T. Coetzee, G. Comi, J. Correale, F. Fazekas, M. Filippi, M. S. Freedman, K. Fujihara, S. L. Galetta, H. P. Hartung, L. Kappos, F. D. Lublin, R. A. Marrie, A. E. Miller, D. H. Miller, X. Montalban, E. M. Mowry, P. S. Sorensen, M. Tintore, A. L. Traboulsee, M. Trojano, B. M. J. Uitdehaag, S. Vukusic, E. Waubant, B. G. Weinshenker, S. C. Reingold and J. A. Cohen, Lancet Neurol., 2018, 17, 162-173.

9 B. R. Brooks, R. G. Miller, M. Swash, T. L. Munsat and D. World, Federation of Neurology Research Group on Motor Neuron, Amyotrophic Lateral Scler. Other Mot. Neuron Disord., 2000, 1, 293-299.

10 B. J. Traynor, M. B. Codd, B. Corr, C. Forde, E. Frost and O. M. Hardiman, Arch. Neurol., 2000, 57, 1171-1176.

11 P. D. Lewis, K. E. Lewis, R. Ghosal, S. Bayliss, A. J. Lloyd, J. Wills, R. Godfrey, P. Kloer and L. A. J. Mur, BMC Cancer, 2010, 10, 640.

12 S. Kar, D. R. Katti and K. S. Katti, Spectrochim. Acta, Part A, 2019, 208, 85-96.

13 S. Roy, D. Perez-Guaita, D. W. Andrew, J. S. Richards, D. McNaughton, P. Heraud and B. R. Wood, Anal. Chem., 2017, 89, 5238-5245.

14 A. Khoshmanesh, M. W. Dixon, S. Kenny, L. Tilley, D. McNaughton and B. R. Wood, Anal. Chem., 2014, 86, 4379-4386.
15 L. Lechowicz, M. Chrapek, J. Gaweda, M. Urbaniak and I. Konieczna, Mol. Biol. Rep., 2016, 43, 1321-1326.

16 M. Paraskevaidi, C. L. M. Morais, K. M. G. Lima, J. S. Snowden, J. A. Saxon, A. M. T. Richardson, M. Jones, D. M. A. Mann, D. Allsop, P. L. Martin-Hirsch and F. L. Martin, Proc. Natl. Acad. Sci. U. S. A., 2017, 114, E7929-E7938.

17 A. L. Mitchell, K. B. Gajjar, G. Theophilou, F. L. Martin and P. L. Martin-Hirsch, J. Biophotonics, 2014, 7, 153-165.

18 C. Lacombe, V. Untereiner, C. Gobinet, M. Zater, G. D. Sockalingum and R. Garnotel, Analyst, 2015, 140, 2280-2286.

19 M. H. Ali, F. Rakib, K. Al-Saad, R. Al-Saady, F. M. Lyng and E. Goormaghtigh, J. Mol. Struct., 2018, 1163, 472-479.

20 D. Yonar, L. Ocek, B. I. Tiftikcioglu, Y. Zorlu and F. Severcan, Sci. Rep., 2018, 8, 1025.

21 M. Paraskevaidi, C. L. M. Morais, D. L. D. Freitas, K. M. G. Lima, D. M. A. Mann, D. Allsop, P. L. MartinHirsch and F. L. Martin, Analyst, 2018, 143, 5959-5964.

22 Y. Y. Broza, L. Har-Shai, R. Jeries, J. C. Cancilla, L. GlassMarmor, I. Lejbkowicz, J. S. Torrecilla, X. Yao, X. Feng, A. Narita, K. Mullen, A. Miller and H. Haick, ACS Chem. Neurosci., 2017, 8, 2402-2413.

23 F. Sedel, C. Papeix, A. Bellanger, V. Touitou, C. LebrunFrenay, D. Galanaud, O. Gout, O. Lyon-Caen and A. Tourbah, Mult. Scler. Relat. Disord., 2015, 4, 159-169.

24 A. Tourbah, C. Lebrun-Frenay, G. Edan, M. Clanet, C. Papeix, S. Vukusic, J. De Seze, M. Debouverie, O. Gout, P. Clavelou, G. Defer, D. A. Laplaud, T. Moreau, P. Labauge, B. Brochet, F. Sedel, J. Pelletier and M.-S. S. group, Mult. Scler., 2016, 22, 1719-1731.

25 L. Breiman, Mach. Learn., 2001, 45, 5-32.

26 I. H. Witten, E. Frank, M. A. Hall and C. J. Pal, Data Mining: Practical machine learning tools and techniques, Morgan Kaufmann, 2016.

27 R. Ihaka and R. Gentleman, J. Comput. Graph. Stat., 1996, 5, 299-314.

28 I. U. Rehman, Z. Movasaghi and S. Rehman, Vibrational spectroscopy for tissue analysis, CRC Press, Boca Raton, 1st edn, 2012.

29 A. Barth, Biochim. Biophys. Acta, 2007, 1767, 1073-1101.

30 L. T. Vu and R. Bowser, Neurotherapeutics, 2017, 14, 119134.

31 J. Beck, H. B. Urnovitz, M. Saresella, D. Caputo, M. Clerici, W. M. Mitchell and E. Schutz, J. Mol. Diagn., 2010, 12, 312-319. 